www.jmscr.igmpublication.org

Impact Factor 5.244

Index Copernicus Value: 83.27

ISSN (e)-2347-176x ISSN (p) 2455-0450

crossref DOI: _https://dx.doi.org/10.18535/jmscr/v4i11.16

Journal Of Medical Science And Clinical Research

\title{
A Descriptive MRI Analysis of Pattern, Location and Grading of Meniscal Injuries of the Knee Joint with Arthroscopic Correlation
}

Authors

\section{Dr Gopinath. G' ${ }^{1}$, Prof. Dr Adaikkapan. $\mathbf{M}^{2}$, Prof. Dr Senthilnathan. $A^{3}$, Dr Rajadirajan. $\mathbf{S}^{4}$, Dr Sankar. $\mathbf{M}^{5}$}

${ }^{1}$ Post Graduate Student, Department of Radiodiagnosis, RMMCH, Annamalai University, Chidambaram 608 002,Tamil Nadu, India

${ }^{2}$ Professor and Head of the Department, Department of Radiodiagnosis, RMMCH, Annamalai University, Chidambaram - 608 002, Tamil Nadu, India

${ }^{3}$ Professor, Department of Orthopaedics, RMMCH, Annamalai University, Chidambaram - 608 002, Tamil Nadu, India

${ }^{4}$ Lecturer, Department of Radiodiagnosis, RMMCH, Annamalai University, Chidambaram - 608 002, Tamil Nadu, India

${ }^{5}$ Post graduate Student, Department of Radiodiagnosis, RMMCH, Annamalai University, Chidambaram 608 002, Tamil Nadu, India

Corresponding Author

\section{Dr Gopinath. G}

Post Graduate Student, Department of Radiodiagnosis, RMMCH, Annamalai University, Chidambaram 608 002, Tamil Nadu, India

\section{ABSTRACT}

Background: Within a decade of its clinical introduction, magnetic resonance imaging has revolutionized diagnostic imaging of the knee. It has become the imaging test of choice for meniscal pathologies in the knee. Purpose: The objective of this study is to correlate the MRI findings with the arthroscopic findings of meniscal injuries of the knee as well as to analyze the type and grade of meniscal tears.

Materials and Methods: MR imaging studies of the knee were performed in 50 clinically suspected patients using a 1.5T MR machine. Various sequences in coronal, sagittal and axial planes were obtained to evaluate the ligaments, menisci, joint effusion and bony contusions.

Results: Medial meniscal tear was seen in $46 \%$ of cases and lateral meniscal tear was found in $38 \%$ of cases. Medial meniscal tear were found in $61 \%$ of cases, who had complete ACL tear. Next common ligament to be torn was the medial collateral ligament, which was seen in 14 patients. $57 \%$ of these patients had grade 2 tear.

Conclusion: MRI is an accurate, non-invasive technique in detecting the meniscal injuries of the knee. It has great capability in classifying them into patterns and grades and can also avoid unnecessary arthroscopic examination. Various sequences are used to identify the tears but certain specific sequences proved to be most useful and should be included as a part of the standard protocol. 


\section{AIMS AND OBJECTIVES}

The objective of this study is to correlate the MRI findings with the arthroscopic findings of meniscal injuries of the knee as well as to analyze the pattern, location and grades of meniscal tears.

\section{MATERIALS AND METHODS}

MR imaging studies of the knee were performed in 50 clinically suspected patients using a $1.5 \mathrm{~T}$ MR machine. Various sequences in coronal, sagittal and axial planes were obtained to evaluate the ligaments, menisci, joint effusion and bony contusions.

\section{DATA ANALYSIS AND RESULTS}

The findings of MRI were compared with arthroscopy to detect the sensitivity, specificity, PPV, NPV, false positive rate and false negative rate of MRI. A total of 50 patients with clinically suspected meniscus injuries were selected. Chisquare test of association is computed apart from sensitivity and specificity analysis to compare the MRI and arthroscopy findings. Frequency distribution statistics is computed for all the outcome variables. The entire analysis is performed using statistical packages of social sciences (SPSS-21).

Table - 1: Age Distributions

\begin{tabular}{|l|l|l|l|l|}
\hline Age & Number & Percentage & Mean & S.D. \\
\hline $18-28$ & 15 & 30 & & \\
\hline $29-38$ & 15 & 30 & & \\
\cline { 1 - 3 } $39-48$ & 11 & 22 & \multirow{2}{*}{36.76} & 11.82 \\
\cline { 1 - 3 } $49-58$ & 7 & 14 & & \\
\cline { 1 - 2 } & 2 & 4 & & \\
\hline Total & 50 & 100 & & \\
\hline
\end{tabular}

Age distribution of the patients is presented in Table -1 .

18 to 28 years and 29 to 38 years are the common age group of the patients. $30 \%$ are observed in both these age categories.

The next common age is 39 to 48 years in which $28 \%$ are observed.

The mean age of the study patients is $36.76 \pm$ 11.82 years.
Fig. - 1 : Age Distributions

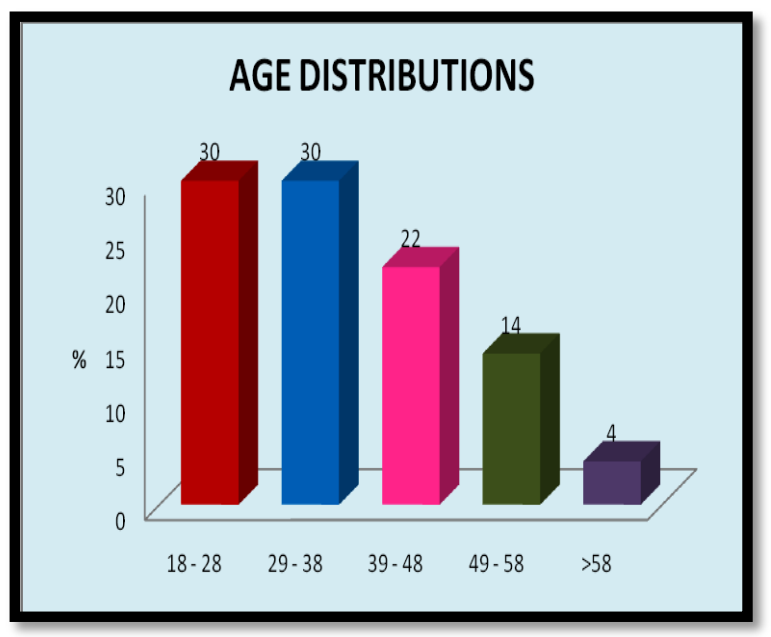

Table - 2: Gender Distribution

\begin{tabular}{|l|l|l|}
\hline Gender & Number & Percentage \\
\hline Male & 39 & 78 \\
\hline Female & 11 & 22 \\
\hline Total & 50 & 100 \\
\hline
\end{tabular}

Table -2 shows gender distribution of the study patients.

Most of the study patients $(78 \%)$ are male participants.

Female constitutes $22 \%$.

Fig. - 2 : Gender Distribution

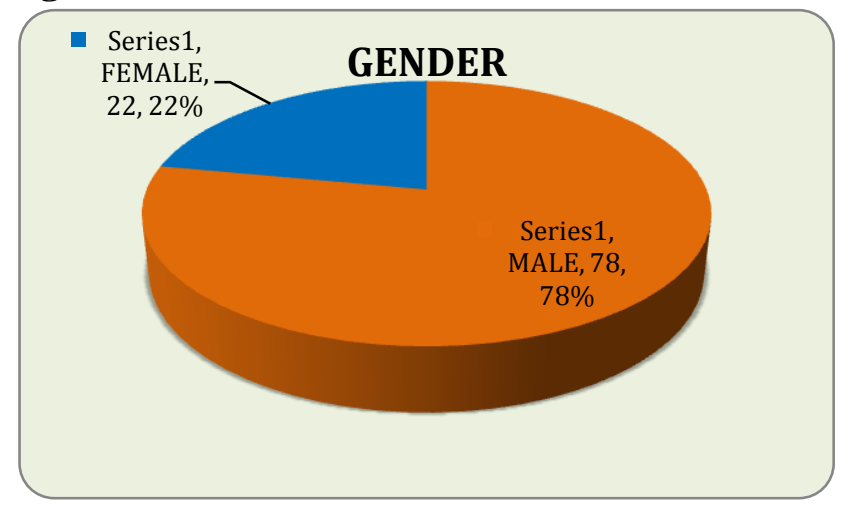

Table - 3: Distribution of Grades of Collateral and Meniscus Tears: MRI Findings

\begin{tabular}{|l|l|l|l|l|l|l|l|l|}
\hline \multirow{2}{*}{ Grade } & \multicolumn{2}{l|}{ MCL } & \multicolumn{2}{l|}{ LCL } & \multicolumn{2}{l|}{ MM } & \multicolumn{2}{l|}{ LM } \\
\cline { 2 - 9 } & $\mathrm{N}$ & $\%$ & $\mathrm{~N}$ & $\%$ & $\mathrm{~N}$ & $\%$ & $\mathrm{~N}$ & $\%$ \\
\hline G1 & 2 & 16.67 & 3 & 20 & 4 & 17.39 & 3 & 15.79 \\
\hline G2 & 6 & 50 & 8 & 53.33 & 3 & 13.04 & 4 & 21.05 \\
\hline G3 & 4 & 33.33 & 4 & 26.67 & 16 & 69.56 & 12 & 63.16 \\
\hline Total & 12 & 100 & 15 & 100 & 23 & 100 & 19 & 100 \\
\hline
\end{tabular}


The grade distribution of collateral and meniscal tears is presented in Table -3 . The most common grade of MCL tear is grade-2 (50\%). About 33.3\% of MCL injury patients have grade 3 tear.

The majority of LCL tear have grade-2 (53.3\%). About $26.67 \%$ of patients with LCL injuries have grade-3 tear.

The most of MM injuries are grade-3 (69.56\%) and again most of LM injuries have grade-3 (63.16) tears.

Fig. 3 - Distribution of Grades of Collateral and Meniscus Tears: MRI Findings

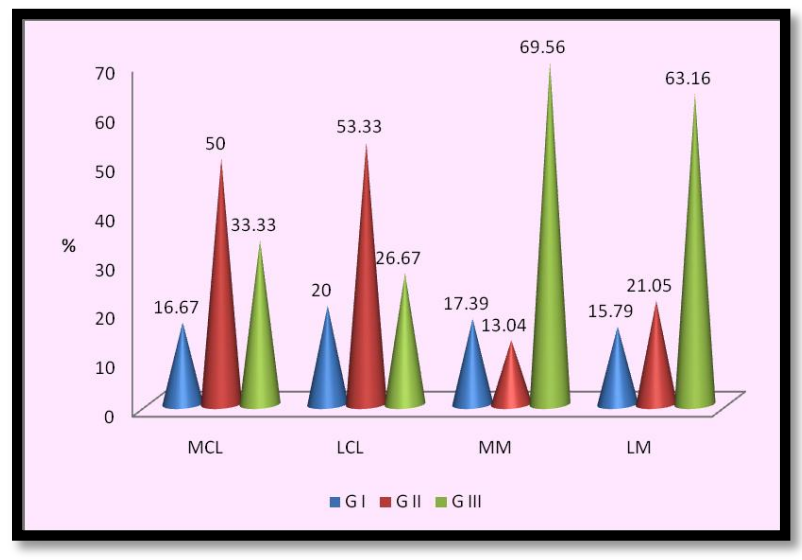

Table - 4: Grades of MM and LM Injuries : Arthroscopy

\begin{tabular}{|l|l|l|l|l|}
\hline \multirow{2}{*}{ Grade } & \multicolumn{2}{l|}{ MM } & \multicolumn{2}{l|}{ LM } \\
\cline { 2 - 5 } & $\mathrm{N}$ & $\%$ & $\mathrm{~N}$ & $\%$ \\
\hline G3 & 15 & 100 & 12 & 100 \\
\hline Total & 15 & 100 & 12 & 100 \\
\hline
\end{tabular}

All the persons (100\%) with MM injuries are grade 3 involvement and again all the patients $(100 \%)$ with LM injuries are grade -3 involvements.

Table - 5: Comparison of MRI Vs Arthroscopy in MM Injuries

\begin{tabular}{|l|l|l|l|l|l|l|}
\hline \multirow{2}{*}{ MRI } & \multicolumn{2}{|l|}{ Yes } & \multicolumn{2}{l|}{ No } & \multicolumn{2}{l|}{ Total } \\
\cline { 2 - 7 } & $\mathrm{N}$ & $\%$ & $\mathrm{~N}$ & $\%$ & $\mathrm{~N}$ & $\%$ \\
\hline Yes & 13 & 86.7 & 2 & 13.3 & 15 & 100 \\
\hline No & 2 & 5.7 & 33 & 94.3 & 35 & 100 \\
\hline Total & 15 & 30 & 35 & 70 & 50 & 100 \\
\hline
\end{tabular}

Chi-Square Test

\begin{tabular}{|l|l|l|}
\hline & Value & 'P' Value \\
\hline Chi-square Value & 32.77 & 0.001 \\
\hline
\end{tabular}

\begin{tabular}{|l|l|}
\hline Comparisons & $\%$ \\
\hline Sensitivity & 86.67 \\
\hline Specificity & 94.29 \\
\hline Positive Predicted Value (PPV) & 86.67 \\
\hline Negative Predicted Value (NPV) & 94.29 \\
\hline False Positive Rate & 5.71 \\
\hline False Negative Rate & 13.33 \\
\hline
\end{tabular}

The comparison shows that, $86.7 \%$ are demonstrated positive MM injuries in both MRI and Arthroscopy (true positive cases). True negative cases are $94.3 \%$. False positive cases are $13.3 \%$ and false negative cases are $5.7 \%$. The chisquare test of association is significant $\left(\chi^{2}=\right.$ 32.77, $\mathrm{P}=0.001)$. Therefore, the findings of MRI and arthroscopy are significantly matching.

The sensitivity of MRI in detecting MM injury is 86.67 whereas specificity is $94.29 \%$. The PPV is $86.67 \%$ and NPV is $94.29 \%$. False positive rate $(5.71 \%)$ is comparatively less than false negative rate $(13.33 \%)$.

Table - 6: Comparison of MRI Vs Arthroscopy in LM Injuries

\begin{tabular}{|l|l|l|l|l|l|l|}
\hline \multirow{2}{*}{ MRI } & \multicolumn{2}{|l|}{ Yes } & \multicolumn{2}{l|}{ No } & \multicolumn{2}{l|}{ Total } \\
\cline { 2 - 7 } & $\mathrm{N}$ & $\%$ & $\mathrm{~N}$ & $\%$ & $\mathrm{~N}$ & $\%$ \\
\hline Yes & 11 & 91.7 & 1 & 8.3 & 12 & 100 \\
\hline No & 1 & 2.6 & 37 & 97.4 & 38 & 100 \\
\hline Total & 12 & 24 & 38 & 76 & 50 & 100 \\
\hline
\end{tabular}

Chi-Square Test

\begin{tabular}{|l|l|l|}
\hline & Value & 'P' Value \\
\hline Chi-square Value & 36.64 & 0.001 \\
\hline
\end{tabular}

\begin{tabular}{|l|l|}
\hline Comparisons & $\%$ \\
\hline Sensitivity & 74.67 \\
\hline Specificity & 84.37 \\
\hline Positive Predicted Value (PPV) & 69.67 \\
\hline Negative Predicted Value (NPV) & 94.37 \\
\hline False Positive Rate & 2.63 \\
\hline False Negative Rate & 8.33 \\
\hline
\end{tabular}

Table -6 shows that, true positive cases for LM injuries is $91.7 \%$. False positive cases are $8.3 \%$ and false negative cases are $2.6 \%$. The chi-square test of association is significant $\left(\chi^{2}=36.64, \mathrm{P}=\right.$ 0.001). Hence the findings of MRI and arthroscopy are significantly matching with each other. 
The sensitivity of MRI in LM injuries is $81.69 \%$ whereas its specificity is $74.37 \%$. The PPV and NPV are $69.67 \%$ and $94.37 \%$ respectively. False positive rate is $2.63 \%$ and false negative rate is $8.33 \%$.

Table - 7: Type of MM and LM Tears

\begin{tabular}{|l|l|l|l|l|}
\hline \multirow{2}{*}{ Type } & \multicolumn{2}{|l|}{ MM } & LM \\
\cline { 2 - 5 } & $\mathrm{N}$ & $\%$ & $\mathrm{~N}$ & $\%$ \\
\hline Oblique & 5 & 31.25 & 3 & 25.00 \\
\hline Horizontal & 2 & 12.50 & 2 & 16.67 \\
\hline Vertical & 4 & 25.00 & 2 & 16.67 \\
\hline Radial & 2 & 12.50 & 2 & 16.67 \\
\hline Complex & 1 & 6.25 & 2 & 16.67 \\
\hline Bucket Handle & 2 & 12.50 & 1 & 8.33 \\
\hline Total & 16 & 100.00 & 12 & 100.00 \\
\hline
\end{tabular}

Oblique type of tears is common in MM (31.25) as well as LM (25\%) injuries. $25 \%$ of patients with $\mathrm{MM}$ injuries are vertical tears. Each of $16.67 \%$ of LM tears has horizontal, vertical and radial type of tears.

Fig. 6 - Type of MM and LM Tears

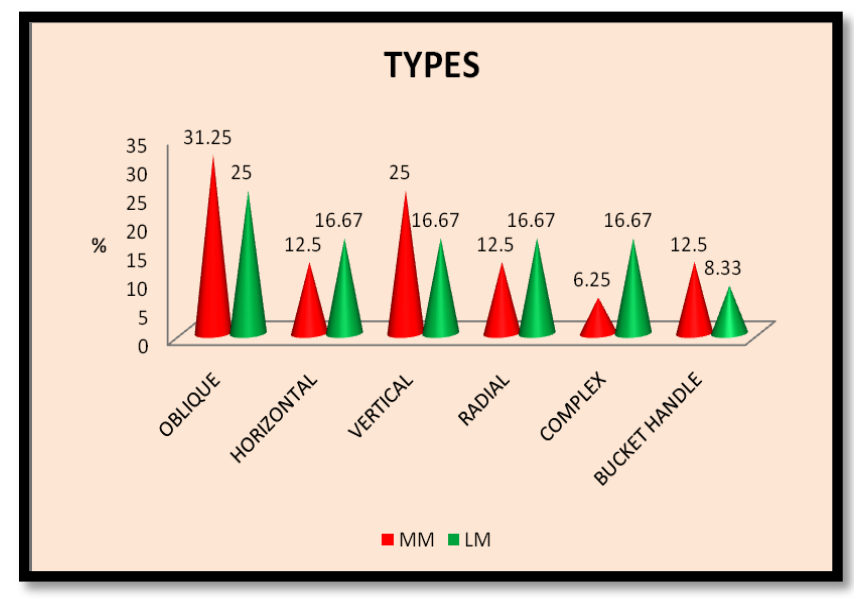

Table - 7: Location of Meniscus Tears

\begin{tabular}{|l|l|l|l|l|}
\hline \multirow{2}{*}{ Location } & \multicolumn{2}{|l|}{ MM } & \multicolumn{2}{l|}{ LM } \\
\cline { 2 - 5 } & $\mathrm{N}$ & $\%$ & $\mathrm{~N}$ & $\%$ \\
\hline Ant. Root & 0 & 0 & - & - \\
\hline Ant. Horn & 2 & 12.5 & 3 & 25 \\
\hline Body & 10 & 62.5 & 7 & 58.33 \\
\hline Post. Root & 3 & 18.75 & 1 & 8.33 \\
\hline Post Horn & 7 & 43.75 & 6 & 50 \\
\hline
\end{tabular}

The location of Meniscus tears shows that, body is the common site of tears in MM $(62.5 \%)$ as well as LM $(58.33 \%)$ injuries. The next common site of Meniscus tears are posterior horn (MM $43.75 \%, \mathrm{LM}-50 \%)$ and anterior horn (MM $12.5 \%, \mathrm{LM}=25 \%)$.
Fig. 7 - Location of Meniscus Tears

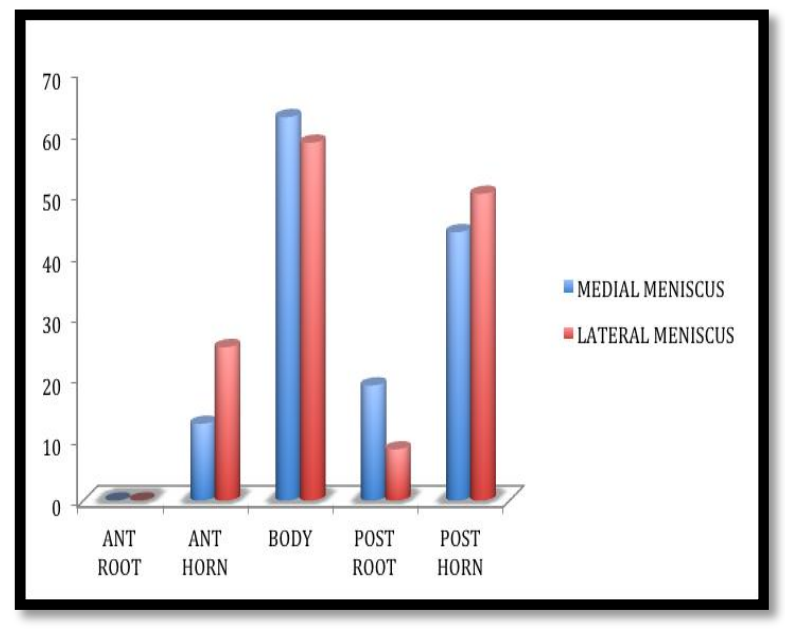

\section{DISCUSSION}

Imaging of the knee presents a special challenge because of its complex structure. A variety of imaging modalities are currently used to evaluate knee abnormalities. These modalities include standard radiography, scintigraphy, computed tomography, magnetic resonance imaging and arthrography.

MR imaging has revolutionized knee imaging. It has been compared by various studies between magnetic resonance and arthroscopic findings. These studies validate the role of MR imaging in the clinical arena especially for the evaluation of ligamentous injuries. There are many advantages of MR imaging over other modalities.

This study included 50 patients who were clinically suspected, underwent MRI of the knee joint. All the scans were conducted in the department of Radio diagnosis, Rajah Muthiah Medical College and Hospital, using a 1.5 Tesla Phillipsopen magnet type scanner. It was done during the period from November 2014 September2016. They were done prospectively and using a scan protocol which included $\mathrm{T} 1 \mathrm{w}$, $\mathrm{T} 2 \mathrm{w}$ and Proton Density images in coronal and sagittal planes and Fat saturated and T2w images in coronal and axial images.

The study population consisted patients in the age group of $18-61$ yrs. Maximum number of patients who underwent MRI of the knee for both cruciate and collateral ligaments belonged to the age group of $18-38$ years. This study also 
showed a male preponderance accounting for $78 \%$ of the caseload.

\section{MEDIAL MENISCUS}

A total of 23 patients $(46 \%)$ showed intra substance signal changes for MM injury. Out of the 23,4 cases $(17.39 \%)$ had G-I injury, 3 $(13.04 \%)$ cases had G - II injury and 16 cases (69.56\%) had G - III injury.

Arthroscopy showed 15 cases (30\%) tears of MM. Horizontal / oblique tear was seen in 7 cases (43\%). Vertical tear was seen in 4 cases $(25 \%)$. Radial tear was seen in 2 cases (12.5\%). Complex tear was seen in 1 case $(6.2 \%)$. Displaced Bucket handle tear was seen in 2 cases $(12.5 \%)$.

Most of our patients had tear in the body (10 cases $-62.5 \%)$ and posterior horn (7 cases $-43.75 \%)$ followed by posterior root ( 3 cases $-18.75 \%$ ) and anterior horn ( 2 cases $-12.5 \%$ ).

$86.7 \%$ are demonstrated positive $\mathrm{MM}$ injuries in both MRI and Arthroscopy (true positive cases). True negative cases are $94.3 \%$. False positive cases are $13.3 \%$ and false negative cases are $5.7 \%$. The chi-square test of association is significant $\left(\chi^{2}\right.$ $=32.77, \mathrm{P}=0.001)$. Therefore, the findings of MRI and arthroscopy are significantly matching. The sensitivity of MRI in detecting MM injury is 86.67 whereas specificity is $94.29 \%$. The PPV is $86.67 \%$ and NPV is $94.29 \%$. False positive rate $(5.71 \%)$ is comparatively less than false negative rate $(13.33 \%)$.

\section{LATERAL MENISCUS}

A total of 19 patients (38\%) showed intra substance signal changes for LM injury. Out of the 19,3 cases $(15.79 \%)$ had G-I injury, 4 $(21.05 \%)$ cases had G - II injury and 12 cases (63.16\%) had G - III injury.

Arthroscopy showed 12 cases (24\%) tears of LM. Horizontal / oblique tear was seen in 5 cases $(41.67 \%)$. Vertical tear was seen in 2 cases $(16.67 \%)$. Radial tear was seen in 2 cases (16.67\%). Complex tear was seen in 2 cases (16.67\%). Displaced Bucket handle tear was seen in 1 case $(8.33 \%)$.
Ruth Crawford et al ${ }^{(52)}$ did a study considering arthroscopy as gold standard. He concluded that results of MRI differ for medial and lateral meniscus with accuracy of around $85 \%$.

Most of our patients had tear in the body ( 7 cases - 58.33\%) and posterior horn (6 cases - 50\%) followed by posterior root ( 1 cases $-8.33 \%)$ and anterior horn ( 3 cases $-25 \%$ ).

True positive cases for LM injuries are $91.7 \%$. False positive cases are $8.3 \%$ and false negative cases are $2.6 \%$. The chi-square test of association is significant $\left(\chi^{2}=36.64, \mathrm{P}=0.001\right)$. Hence the findings of MRI and arthroscopy are significantly matching with each other.

The sensitivity of MRI in LM injuries is $91.69 \%$ whereas its specificity is $97.37 \%$. The PPV and NPV are $91.67 \%$ and $97.37 \%$ respectively. False positive rate is $2.63 \%$ and false negative rate is $8.33 \%$.

Ali Akbar EsmailiJah et al ${ }^{(53)}$ compared MRI with arthroscopic findings and calculated the sensitivity (MM - 79\%, LM - 66\%), specificity (MM $-94.7 \%, \mathrm{LM}-86.2 \%$ ), positive predictive value $(\mathrm{MM}-92 \%, \mathrm{LM}-61 \%)$ and negative predictive value (MM - 96.7\%, LM - 92.6\%) for meniscal tears, which is in consistent with our study.

\section{CONCLUSION}

The present study was conducted as a prospective study using a Phillips 1.5 T MRI Scanner in the department of Radiodiagnosis, Rajah Muthiah Medical College and Hospital, Chidambaram aimed to evaluate the usefulness of MRI to detect the ligamentous injuries and meniscal injuries of the knee.

In this study, it was found that

Horizontal tears are the most common tear pattern noted in both Medial and lateral meniscal tear predominantly involving the body and posterior horn.

Majority (46\%) of patients had medial meniscus injury and the rest $38 \%$ of patients had lateral meniscus injury. 
Both ACL and MCL tears showed predilection towards Medial meniscus tear.

Meniscii are best demonstrated on Fat saturated Proton density sagittal sequence.

MRI is an accurate, non-invasive technique in detecting the meniscal injuries of the knee. It has great capability in classifying them into patterns and grades and can also avoid unnecessary arthroscopic examination. Various sequences are used to identify the tears but certain specific sequences proved to be most useful and should be included as a part of standard protocol.

\section{BIBLIOGRAPHY}

1. Keith L Moore, Arthur F Dailey, Clinically Oriented Anatomy- 5th edition, Churchill Livingstone, Edinburgh; 1999

2. Agur AIVIR, Lee MJ. Grants' Atlas of Anatomy, 9t11 edition. Baltimore: Williams and Wilkins; 1991.

3. DeMaeseneerM, Shahabpour M, Van Roy F,et al. MRimaging of the medial collateral ligament bursa : findings in patients and anatomic data derived from $\begin{array}{lll}\text { cadavers. } & \text { AJR }\end{array}$ JRoentgenol2001;177:911-917.

4. Rothstein CP, Laorr A, Helms CA, et al. Sernimembranous - tibial collateral ligament bursitis: MR image findings. AJR Am Roentgenology 1995;155:875-877.

5. Kean DM, Worthingston BS, Preston BJ, et al. Nuclear magnetic resonance imaging of the knee: examples of normal anatomy and pathology. British J Radiol1 983;56(666):3 55-364.

6. $\mathrm{Fu} \mathrm{Fh}$, Hamer $\mathrm{CD}$, Vince KG. Knee Surgery, Vol 1 Baltimore (BM): Williams and Wilkins; 1994.

7. Prickett, William D, Ward S, Mathew M. magnetic resonance imaging of the knee. Sports medicine vol 31(1 4),2001 ;997-1 019.

8. Crotty JI4, Monu JUV, Pope TL Jr. IMagnetic resonance Imaging of the musculoskeletal system: part 4. The knee. Clinical orthopedics and related research 330:288-303.

9. Hartzman S. Reicher MA, Basset LW, et al. MR imaging of the knee part 2. Chronic disorders. Radiology 1987; 162:553-557.

10. Turner AD, Prodromos C, Petasnick J, Clark JW. Acute injury of the ligaments of the knee: Magnetic Resonance Evaluation 1985;1 54:717-722.

11. Smith Douglas, May David, Philips Paul. MR Imaging of the anterior cruciate ligament: frequency of discordant findings and correlation with arthroscopic findings. Am J Roentgenol 1996;166:41 1-413.

12. Singh JP, Garg L, Shrimalli R, Setia V, Gupta V. MR imaging of the knee with arthroscopic correlation in twisting injuries. IJRI 2004;14(1):33-40.

13. Roberts C, Towers JD, Spangehl, Carrino $\mathrm{J}$, Morisson M/. Advanced MR Imaging of the cruciate ligaments. Magnetic Resonance Imaging Clinics 2007;148:762765.

14. Ruth Crawford, Gayle Walley, Stephen Bridgman, Nicola Maffulli.(2007). "Magnetic resonance imaging versus arthroscopy in the diagnosis of knee pathology, concentrating on meniscal lesions and ACL tears: a systematic review" Br Med Bull (2007) 84 (1): 5- 23.

15. Ali Akbar Esmaili Jah, Sohrav, Keyhani, Reza Zarei. (2005). "Accuracy of MRI in comparison with clinical and arthroscopic findings in ligamentous and meniscal injuries of the knee". Acta Orthop. Belg.,2005, 71,189-196.

16. Munk B, Madsen F, Ludorf. (1998). "Clinical magnetic resonance imaging and arthroscopic findings in knees: A comparative prospective study of meniscus, ACL and Cartilage lesions". March 1998, Vol14(2): 171-175. 